\title{
Development of Free Electron Lasers in Europe Local and Global Implications - 2016
}

\author{
Ryszard S. Romaniuk
}

\begin{abstract}
Free electron laser FELs are built in Europe mainly as nondependent infrastructures, or as a development of synchrotron ones. They are constructed mainly in centres which have considerable experience with synchrotron light sources of the third generation like DESY, Trieste, INFN, etc. Advances in very energetically efficient superconducting linear accelerators for electron beams, like TESLA type, caused an abrupt development of FEL machines all over Europe. New generation of FELs emits light beam of extreme intensity, good parameters, in IR, VIS, UV, EUV and X-ray spectral regions. The machine construction teams comprise also of young active researchers from Poland. In particular, these is a considerable participation of M.Sc. and Ph.D. students from Warsaw University of Technology at building of FLASH I, FLASH II, and EXFEL machines. Unique experiences gathered at work with these large experiments result in development of these young teams, and their further engagement in new initiatives: laser, laser - accelerator, inertial, plasma, plasma - energy, etc. This is what we observe with satisfaction. However, due to the lack of large research infrastructures in Poland, we are not members of the infrastructure owner clubs. Our young researchers may take part in the initiatives only indirectly as members of cooperative teams from the leading countries. As a further consequence, there is also a confined access of Polish laser and accelerator researchers to some kinds of European infrastructure development projects now under realization within the $\mathbf{H 2 0 2 0}$.
\end{abstract}

Keywords : laser technology, laser development, lasers, free electron lasers, photonics, EUV radiation, RTG radiation, research infrastructures

\section{INTRODUCTION}

$\mathbf{T}$ HE idea to use a relativistic electron beam passing previously through a bent magnet and later through a wiggler for generation of coherent EM radiation in the spectral range from IR to UV was presented in literature more than half a century ago [1]. Microwave tube called Ubitron - an early precursor of a FEL was constructed in 60-ties. First fundamental theoretical work on FEL was carried out in 60-ties and 70-ties. The first precursors of a FEL were tested in labs in 1971 for wavelength $10 \mu \mathrm{m}$ [2], and next in 1977 [3]. The SASE theory - self-amplified spontaneous emission of FEL radiation was elaborated in mid-80-ties [4-5]. First solutions of a FEL machine based on ring accumulation, typical for classical synchrotron architecture, thus with a beam of relatively low intensity and almost no coherence. A linear bypass in the circle was used to insert a wiggler (Halbach magnetic matrix), and later undulator. Optical field was amplified in a resonant cavity analogous to the one used in classical optical lasers. Further increase in electron beam energy and shortening of the wavelength of optical beam met a confinement associated with the lack of relevant optical mirrors for $\mathrm{X}$ rays (short wavelengths of few tens of $\mathrm{nm}$ ), even using the technique of grazing angle incidence and reflection and circular resonant cavities. An alternative is to use no resonant cavity in laser architecture, i.e. a single pass solution for the optical beam pulse. A summary of this early work is in [6]. Development in the construction of linear accelerators and RF electron guns with a photocathode excited by a laser pulse, and ultra-precise long undulators, resulted in larger intensities of electron and photon beams, during a single pass of the pulse.

The photocathode RF electron gun provides short, picosecond pulses of a considerable charge above $1 \mathrm{nC}$ and small normalized emittance in the range of mm-mrad. First solutions resulted in an IR beam. Visible range FEL working with 390 $\mathrm{nm}$ was launched first time in ANL [7] in 2001, and soon afterwards in DESY in VUV region $998 \mathrm{~nm}$ in 2002 [8]. Laser sources with linear accelerators turned out to be completely different from previous solutions, leading to unprecedented parameters like intensity, coherence, beam quality, wavelength, and pulse duration [9-18]. They were named as light sources of the fourth generation. Today the FEL community starts to speak of the birth of future light sources of the fifth generation. They are compact, attosecond, ultra-bright, quantum FELs with integrated MEMS superconducting or plasma photonic undulators, powered by new low-charge $(\mathrm{pC})$ plasma-laser and photonic methods of electron beam acceleration.

\section{FEL LASERS IN THE WORLD AND IN EUROPE}

Free electron lasers are under intense development all over the world and in Europe, built as standalone infrastructures, or as extensions to existing large synchrotron machines. Usually they are built in research centres having large experience with synchrotron light sources of the third generation, as in DESY, Trieste, INFN, PSI, etc. [19]. Development of very energy efficient accelerators of TESLA type caused FEL machines to flourish. TESLA FELs generate light beams of high intensity, high quality and spanning the whole spectral region from IR, via VIS, UV, EUV and RTG. FEL peak beam intensity is many orders of magnitude greater than in the synchrotron and is equal for EXFEL to $5 \times 10^{33}[\mathrm{fot} / \mathrm{s} / \mathrm{mm} 2 / \mathrm{mrad} 2 / 0,1 \% \mathrm{BW}]$, and the average intensity is more than four orders of magnitude bigger $\left(10^{25}\right)$. Early development of EUV and RTG liniac based FELs was marked by low repetition frequency of the light pulses. This confinement was caused by injector characteristics, warm accelerator and features of high power MW klystrons. The 
EXFEL under construction with a superconducting linac will have $27 \mathrm{kHz}$ as the maximum X-ray pulse repetition frequency. Future FEL infrastructures now of the fourth, and soon of the fifth generation will be the main tunable sources of the intense, coherent photon beams from the IR to hard X-rays of unprecedented time and spectral resolution. A serious drawback of the fourth generation light sources are their immense dimensions, extreme costs and difficult access to the beam time.

Now the main global FEL infrastructures of the fourth generation, active or under construction, are: FLASH (2005, DESY) [flash.desy.de], EXFEL (2016 DESY) [xfel.eu] combined with CFEL Research Centre, FERMI (2010 INFN) [elettra.trieste.it/ lightsources/ fermi.html], SwissFEL (PSI 2017) [psi.ch/swissfel], SACLA XFEL (2012 RIKEN) [xfel.riken.jp]; PAL-XFEL (2015 Pohang) [pal.postech.ac.kr/paleng/], LCLS-LCLSII (2009, 2017 SLAC) [lcls.slac.stanford.edu]. Apart of these immense, very big or only big machines, there are active in Europe numerous bigger and smaller research FELs, or test facilities for certain parts of FEL infrastructure, run by particular national laser and synchrotron laboratories. There are built complete standalone FELs or their sub-systems like new generation injectors, undulators, compact accelerators, etc. These infrastructures are, for example: FLARE (Nijmegen, Netherlands); CLIO - LCP (Orsay, France); ELBE FEL - HZDR (Germany); ENEA Compact FEL (Frascati, Italy); SPARC FEL Frascati INFN; FHI FEL (Fritz-Haber Institute, Germany); FELIX - FOM (Rijnhuizen, Netherlands); IR-FEL S-DALINAC (Darmstadt Germany); NovoFEL - Budker Institute (Russia); Tel Aviv University FEL (Israel), University of Twente Cerenkov FEL (Netherlands), and other.

A considerable number of young researchers, physicists and engineers, and Ph.D. students from Poland, are participating in the construction, maintenance and research in the listed European FEL centres. A particularly big input of M.Sc. and Ph.D. students from the Faculty of Electronics and Information Technologies, Warsaw University of Technology was at the design and construction of the following FEL machines: FLASH I, FLASH II, and recently European EXFEL. Experience gathered at the work for such big and complex experiments result in development of professional research groups and their engagement in several related new laser, laseraccelerator, accelerator, HEP instrumentation, inertial fusion, high temperature plasma, and energy-plasma initiatives. Instrumentation character for such experiments is similar at various levels. And this is just the case. Unfortunately, due to the lack of a large laser and HEP research infrastructure in Poland, we are not the members of the prestigious owner clubs, and our researchers may participate in the work of these clubs indirectly, as members of the international groups. As a result, the access to certain kinds of the European infrastructure development projects is somehow, or at least partly, confined. Despite of this the national FEL and accelerator community tries to participate practically in all European activities via relevant institutional engagement and via scholarships for young researchers.

FEL infrastructures of the fourth generation are developed in Europe mainly as initiatives of large laboratories. Apart of that, there are initiated relevant consortia, collaborations, research projects - now inside the $\mathrm{H} 2020$, infrastructural projects, synergy networks, machine user training, machine operator training, etc. An initiator of numerable actions in this area is the DESY FEL Research Centre - CFEL. The initiators are also European Research Networks - IRVUX FEL, LaserLabEurope, FELs of Europe, EuroFEL, and European XFEL. In particular, these initiatives concern, what is essential for technology development, the establishment of common research projects under the EU umbrella, previously inside the FP6 and FP7, now under H2020, and under FP9 in the future.

The European community of large research infrastructures in high power, high energy lasers and laser-accelerators is very active and takes care very efficiently of their interests. This community realizes now many projects financed by various sources: institutional, national, international, multilateral, industrial, and European H2020. We participate, as a national research community, and as a country only marginally in many of these initiatives, due to a lack of national research infrastructures of a relevant European scale weight. It is a high time to wake up and break this vicious circle of inability and country research marginalization. International involvement of the national science which requires essential support of the government is not proportional to the potential abilities and ambitions of Poland and the Polish research community. Discovery ambitions of large scale, igniting young researchers equally effectively around the world, our young engineers and physicists have to realize abroad. Poland has to build a large research infrastructure, of the European and discovery class, which will actively attract young researchers from Poland and from abroad. Large laser infrastructure, of discovery class, like FLASH, EXFEL, LCLS etc, has a profound impact on the whole global research communities [22-23], not only laser ones but also in many other research branches like biology and medicine, material engineering, chemistry and many more.

\section{EUROPEAN FEL LASER CONSORTIA}

EUCALL - European Cluster of Advanced Light Sources [eucall.eu] is a networking activity financed by the H2020 during the time span 2015-2018, which groups the owners and key users of the main large-scale infrastructures and user facilities, including FELs, synchrotron, laser and accelerators, like EXFEL, DESY, Elettra, ELI, ESRF, HZDR, Lund University, PSI, and two networks LaserLab-Europe and FELs of Europe. The European Cluster of Advanced Light Sources was initiated by DESY in October 2015. The aim of EUCALL is interaction of all participants to prepare common research methodologies, search for common discovery possibilities, development of common tools supporting further cooperation of the largest infrastructures in the future. With some regret, unrest and anxiety it is to be noted that Poland is not a member of the EUCALL, which is a consequence, among others, of the lack of our direct involvement, some time ago, in such initiatives as ELI, HIPER, CANN, etc. Work packages of EUCALL are strictly focused on a considerable strengthening of the cooperation between the major European laser infrastructures. 
Inside EUCALL, there are operating work packages and user consortia for FEL infrastructures. SIMEX work package develops and implements fundamental simulation platform for the users and infrastructure operators, to enable a full simulation of each experiment with various light sources. The simulations trace photons from the source, via optics and interaction region, to the detector. Researched samples embrace such objects as weakly dissipating biomolecules, strong interaction of radiation with matter combined with density modulation of matter, dynamic compression of matter to the densities of planetary cores, etc. The work package UFDAC develops hardware and software technologies for ultra-fast online data acquisition. Data include on-line image processing, data injection and transfer, data digitization and processing for laser experiments with fast pulse repetition and ultra-short impulses. HIREP develops common, normalized technologies of fast delivery, exchange and removal of the samples from the interaction region, and fast decentralized sample characterization. HIREP aims at considerable increase of the access to the experiments, and in particular to the interaction time, by numerable users. This leads through a complete change in the technique of sample manipulation. PUCCA develops control and characterization technologies of individual X-ray laser pulses. PUCCA works on high-resolution, femtosecond individual characterization methods for each pulse separately, in such a way as not to change the pulse parameters. There are used PAM - Pulse Arrival Monitors and BPM - Beam Position Monitors, wave-front sensors, transparent beam intensity monitors, and analytical software. SYNERGY work package seeks new research possibilities in the area of advanced laser techniques, in particular with active participation of users.

EuroFEL or European XFEL Consortium was established in 2012, as an agreement supporting research in the area of XFEL machines and building of FEL infrastructures in Europe [eurofel.org], [eurofel.eu]. Building of the Consortium was supported by an European project of the preparatory phase character - IRVUX-PP [irvux.eu]. Under the same name, there was realized an European project coordinated by DESY of the design study character, which was successfully finished in 2007. A member of the European XFEL Consortium from Poland is NCBJ. A great advantage of the membership in this organization for an institution from Poland is the possibility to participate in other European initiatives despite the lack of relevant active, local FEL infrastructure.

UMBRELLA Consortium was signed in August 2015 by the European owners of the light and neutron sources: ALBA, DESY, Diamond Light Source, Elettra, EMBL Heidelberg, ESRF, EXFEL, HZB, ILL, IAS, KIT, PSI, STFC, SOLEIL. Umbrella is a pan-European integrated IT platform servicing the European large-scale research infrastructures. Umbrella includes data bases for personnel, resources, media, research methods, etc. It was initiated by the European project IRVUXPP.

FELS OF EUROPE is an initiative of such ESFRI projects as EuroFEL and European XFEL [fels-of-europe.eu]. The members are all owners of the FEL infrastructures working or under development in Europe, EXFEL partners, IRFELs and Turkish FEL laser project TARLA. Together there are 14 partners from 10 countries, including Poland. FELSoE was established as a permanent organization of long-term cooperation, independently of external financing by European programs. The aim of the Collaboration is optimization of the usage of European FEL infrastructures and know-how intellectual resources in this area, so as to increase the competence and competitiveness of Europe in research, applications and industry, as well as provide access to laser infrastructures by much larger research and technical communities than it is today. The Consortium FELs of Europe is an initiative renewing and integrating previous European activities in this area. It is a trial to embrace with a voluntary coordination of much larger activity area than before - apart from research and technical, also training of experts, education, strong support for users, also lobbying and outreach activities, dissemination and interaction with the society, and promoting biological, medical and industrial applications.

All big FEL infrastructures build, sometimes even formally, the user consortia. This stems from the fact that generally the access to the laser beam is difficult, and the beam time is very expensive. The access is allotted by various methods, either by proprietary contracts with the owners of experimental stations in the infrastructure main experimental hall, via dedicated grants, or creation of topical research groups, etc. Each of the lasers has usually its own well organized groups of users. They are organized around specific parts of the infrastructure and particular experiments. Such user consortia working with the FLASH and EXFEL laser infrastructures are listed and succinctly characterized below.

FLASH laser infrastructure has evolved as a result of the development of superconducting accelerating technology and TESLA Nb cavities of extremely high finesse, and also Tesla Test Facility TTF experiments. FLASH laser was assumed to be a pilot solution for the design of ten times bigger European X-Ray FEL, or EXFEL. FLASH generates light transversely coherent using SASE mechanism, of the fundamental wavelength in the range $4-52 \mathrm{~nm}$. It generates also the third and the fifth harmonic wave. It serves as a user and machine study facility since 2005. Length of the infrastructure is $260 \mathrm{~m}$. Till 2009 it was the only FEL infrastructure in the world to generate very intense EM wave in the range of soft X-rays.

Due to an immense interest of the users, the FLASH infrastructure was supplemented later by additional laser FLASH II with a second undulator and a separate experimental hall. Average energy of a single pulse is in the range $10-500$ $\mu \mathrm{J}$. Time duration of a pulse is in the range $45-200 \mathrm{fs}$. Pulse repetition frequency is in the range $10 \mathrm{~Hz}-5 \mathrm{kHz}$, what gives maximum pulse power in the range $1-3 \mathrm{GW}$ and average power $600 \mathrm{~mW}$. Spectral width of a pulse is $0,3-2 \%$. Each pulse contains from $10^{11}$ to $10^{13}$ photons. Average luminosity is in the range $10^{11}-10^{13}$, and maximum $10^{29}-10^{31}$ [fot $/ \mathrm{s} / \mathrm{mrad} 2 / 0,1 \% \mathrm{bw}$. Irradiance of the focused beam is over $10^{16} \mathrm{~W} / \mathrm{cm}^{2}$.

One of the most important features of FLASH laser is high intensity beam availability in the long-wavelength part of the water window, spanning in the region $2,3-4,4 \mathrm{~nm}$. Water is transparent in this region for the radiation, which in turn is strongly absorbed by carbon atoms. FLASH enables contrast 
investigations of biological samples for these wavelengths in water solutions.

Energy source for the laser is a superconducting electron linear accelerator, consisting of 7 modules each with 8 niobium cavities, of TESLA type. The cavities work at standard frequency $1,3 \mathrm{GHz}$, beam energy is from $350 \mathrm{MeV}$ to $1,25 \mathrm{GeV}$. The liniac uses a compound photocathode laser and RF electron gun of a small emittance, which is required for an efficient SASE process. FLASH is a laser without a resonator, of a single passage of high intensity electron beam. Maximal current intensity is 1-2 A, and this high value is required by the undulator working in the area of high gain and saturation. High intensity of the peak current is obtained in a compressor of long electron pulses, with medium electron beam energy level of 150 $-450 \mathrm{MeV}$. The undulator, $27 \mathrm{~m}$ long, consists of permanent $\mathrm{NdFeB}$ magnets of constant gap $12 \mathrm{~mm}$, and 27,3 $\mathrm{mm}$ period, and maximum magnetic field 0,47 $\mathrm{T}$. The undulator induces micro-bunching of the electron pulsed beam. Micro-bunches radiate coherently in the range of X-rays. The generated X-ray beam is transmitted to the experimental hall.

FLASH II laser is an essential extension of the FLASH I laser infrastructure. It has an additional experimental hall doubling the number of user stations, and a separate tunnel with undulator of a changeable gap (as opposite to constant gap in FLASH I undulator). Due to this geometry, it is possible to deliver simultaneously two nondependent wavelengths to the experimental stations. The electron beam is switched from the TESLA linac at a very small angle of $1^{\circ}$ between the constant gap undulator of FLASH I and a changeable gap undulator of FLASH II. The infrastructure provides up to the fifth harmonic of the fundamental wavelength of $0,8 \mathrm{~nm}$. Low-loss optical components are used, optimal for this wavelength. FLASH II uses the laser seeding technique for the wavelengths $10-40 \mathrm{~nm}$ (as opposite to SASE technique in FLASH I). The seed laser is Ti:Sa of repetition frequency $100 \mathrm{kHz}$. For longer wavelengths there is used SASE technique. FLASH II infrastructure also enables tests with a high power $\mathrm{THz}$ source with dedicated undulator.

FERMI@Elettra FEL complex (Free Electron Laser radiation for multidisciplinary applications) is a two laser source of the fourth generation built at the synchrotron infrastructure of the third generation ELETTRA in Trieste. FERMI works with optical seeding and generates pulsed wave - time 10-100 fs, wavelength $4-100 \mathrm{~nm}$, VUV, EUV and soft $\mathrm{X}$-rays. Wavelength $4 \mathrm{~nm}$ is obtained from the first harmonic. Tuning of the first harmonic spans the spectrum 20-100 nm. The generated pulsed radiation is transversely and longitudinally coherent. The applied undulators of APPLE II type allow to obtain linear polarization in both directions vertical and horizontal, and also circular in both directions. Beam generation with optical seeding, as opposing to SASE, guarantees very good properties of time coherence, flexibility of tuning in the wavelength and polarization domains. An advantage of the seeding method is the ability to shorten considerably the undulator as compared to SASE, and large spectral resolution enabling resignation from the monochromator. Seeding signal is provided from a classical optical, pulsed, high power laser working with $260 \mathrm{~nm}$. Seeding signal is propagated co-linearly with the electron beam throughout the whole length of the undulator.

The frequency of optical pulses is synchronized with the frequency of the electron bunches, in such a way that both pulses optical and electron cover each other. A complex and multifunctional undulator is divided to three parts: modulation, chromatic dispersion and radiation. The electric field of the laser beam, in modulation part, modulates the energy of transversely shifting electron beam with its own frequency. A subtle energy modulation is transformed to space distribution of charge density in the bunch during its passage through magnetic, chromatic dispersion filter. The resulting micromodulation of bunch density has higher harmonics originating from the wavelength of the seeding laser. The radiation part of the undulator, with tunable magnetic field intensity, chooses the fundamental wavelength or harmonic wavelength during a single passage of the signal. Duration time of the FEL optical pulse is approximately equal to the pulse from the seeding laser, and depends on the polarization properties of this part of the undulator.

To generate the wavelengths in the area of water window, or to reduce the wave from $20 \mathrm{~nm}$ to $4 \mathrm{~nm}$, the FERMI infrastructure possesses a second separate two-stage laser line consisting of a HGHG cascade of high gain for harmonics generation. The first stage of the second line is similar to the first one generating the wave $20 \mathrm{~nm}$, but has shorter radiation part of the undulator and is seeded with a shorter wavelength $210 \mathrm{~nm}$, and amplifies harmonic wave $10 \mathrm{~nm}$. The modulator of the second stage is tuned to the radiator output of the first stage. The second modulating stage of the undulator modulates again the electron bunch in energy and next density. The second radiator is tuned to the harmonic wave of the first stage in the second line. The output wavelength is shortened four times in this way. The second laser cascade line has also a magnetic delay line between both stages to improve laser efficiency and pulse quality. The fotoinjector bases on a classical microwave solution with a 1,5 resonant cavity. The pulses are $10 \mathrm{ps}$, with repetition frequency $10-50 \mathrm{~Hz}$, charge $1 \mathrm{nC}$, and mean root square normalized transverse emittance approximately $1 \mathrm{~mm}$ rad for $100 \mathrm{MeV}$. The fotoinjector with an RF gun has two RF sections. The liniac works with frequency $3 \mathrm{GHz}$. Liniac sections are separated by bunch compressors. Maximum beam energy is $1,5 \mathrm{GeV}$. Focusing system of liniac is optimized in order to minimize the transverse emittance caused by transverse wakefields, dispersion of the momentum and coherent synchrotron radiation in the beam path curvatures.

FERMI laser is equipped in a complex distribution system for laser beam distribution, which is behind undulators and in front of the experimental stations. The beam distribution system enables beam status characterization, beam manipulation, noninvasive measurements of beam intensity spectral distribution, position, coherence, polarization, change of beam parameters, beam splitting, delay, focusing, etc. All actions may be done with temporal resolution up to a single pulse, or from pulse to pulse. Beam energy spectrometer measures spectral contents of a single pulse, and is equipped in three diffraction gratings, working in the first diffraction order, thus covering the full spectrum $100-4 \mathrm{~nm}$. The gratings may be used effectively up 
to the third order of diffraction. Beam re-focusing is done by bent flat mirrors in the Kikrpatrick-Baez geometry and a diagnostic circuit with a few wave phase-front sensors, YAG screens, fluorescence screens and trace foils from PMMA and from silicon. The obtained focus has dimensions $5 \times 8 \mu \mathrm{m}^{2}$. Wavefront splitting auto correlator and retarder of the transmitted EUV/SXR beam was applied. One of the split beams is retarded and next again recombined in order to obtain a double pulse of regulated mutual delay in the range 2-30 ps for all wavelengths generated by the laser. The FERMI infrastructure is equipped in a number of optical pulse, high power lasers enabling pulse - probe experiments in all kinds of multi-beam geometry.

SwissFEL is a compact national laser built by the Paul Scherer Institute in Willingen. [psi.ch/swissfel]. Laser will generate 10 fs pulses with repetition frequency $100 \mathrm{~Hz}$, over 6 GW pulse power, spectral range $0,1-7 \mathrm{~nm}$. PSI undertakes great effort according to the ambitions of the research community of this country. Such great initiatives attract young researchers to Switzerland. Around such initiatives there are generated many innovative spin-offs. The planned experiments with the SwissFEL infrastructure are focused around the disciplines which have today and in the near future a strong impact on the Swiss and European economy like: material engineering, chemistry, biology, medicine, space engineering and genetic engineering. A special aspect of PSI activities is the presence of immense CERN in this country and despite of this carrying ambitious, own and decisive research policy.

$\mathrm{X}$-ray laser of very good parameters considerably widens the research field of the PSI. Compact laser bases on innovative technological solutions embracing: electron gun using 2,5 cavity and electronically tuned, high power semiconductor modulators, work in the microwave $\mathrm{C}$ band, vacuum undulator technology of very short magnetic field period, APPLE II technology undulator with full polarization control, and generation of hard X-rays. Application of new technologies enabled lowering of the FEL dimensions several times in comparison with classical solutions. This led in consequence to lowering the costs of the whole machine. The laser has two experimental lines: hard $X$ radiation 1-7A (2017r.), and soft $X$ radiation 7-70A (2018r.), and each line has three experimental stations. Now the work goes on installation of the liniac and undulator infrastructures. Readiness of the liniac and 12 undulators is scheduled for the end of 2016. High quality and coherent beam availability of hard X-rays of controlled polarization state and high intensity is scheduled for 2017, what confirms the highest technological status of the SwissFEL infrastructure in the global scale. PSI is famous from active and efficient actions combined with modern technology transfer to industry. And this is just an example.

Laboratory MAX IV in Lund is an operator of three accumulation rings for synchrotron light sources. The planned MAX IV FEL laser is a supplement of this large infrastructure. Energy source will be an extended to 5-6 GeV existing $3 \mathrm{GeV}$ liniac. The laser will use a lot of the existing and modified infrastructure at the possible low cost of additional investments.

Apart of large infrastructures, there are operated in Europe numerable IR and smaller test FEL facilities. ALICE and
CLARA are test infrastructures operated in STFC Daresbury Science and Innovation Centre. ALICE - Accelerators and Lasers in Combined Experiments is a test infrastructure which contains a fotoinjector with a superconducting booster $350 \mathrm{keV}$ $-8,35 \mathrm{MeV}$, superconducting liniac $30 \mathrm{MeV}$ working in a loop circuit with energy recovery geometry, magnetic pulse compressor, and optical resonant FEL cavity with a wiggler for wavelengths $6-8 \mu \mathrm{m}$. The aim of the infrastructure is research of new technical solutions for FEL lasers working in optical, IR and $\mathrm{THz}$ spectral regions. There are carried out investigations on the use of this infrastructure in cancer research. A compact linear accelerator performed in warm technology CLARA Compact Linear Accelerator for Research and Applications - is an infrastructure for proposed compact solution of a FEL. CLARA uses the existing infrastructure of local synchrotron light source, including a VELA photoinjector.

SPARC - is a test infrastructure built in INFN Frascati. The work concerns new constructions of undulators, in particular high harmonics generation, and seeding techniques. SPARC is used together with a built in this laboratory high power $200 \mathrm{TW}$ laser infrastructure.

LUNEX 5 - is a test infrastructure for future light sources of the fifth generation. It is a project of a compact FEL laser realized by synchrotron centre SOLEIL. It uses new acceleration and light generation technologies in an integrated undulator.

FEL IR - Europe has a numer of active IR FEL facilities. FEL CLIO is localized in LCP-CNRS laboratory at Paris University in Orsay. It generates wavelengths $2-120 \mu \mathrm{m}$. FELBE is working in HZDR laboratory in w Drezden, and generates wavelengths $4-250 \mu \mathrm{m}$. FELIX, FELICE, and FLARE lasers are working in Nijmengen laboratory and generate wavelength $2-1500 \mu \mathrm{m}$. TARLA is a planned FEL infrastructure in Turkey.

\section{FEL ACTIVITIES IN POLAND}

WaFEL and PolFEL lasers were FEL laser designs done bravely, yet visionary in Swierk [polfel.pl] a few years ago. The design aimed at a CW machine. The names originate first from the Warsaw FEL and then the Polish FEL. The original design has based on large experiences of Polish engineers and physicists working in numerable FEL laboratories around the world, or working in such FEL oriented projects as TESLA, TTF - Tesla Test Facility, FLASH - DESY, SPARC-INFN in Frascati, Fermi at ELETTRA, also CEBAF in Jefferson Lab and other [polfel.pl] [20-21]. Poland has relevant, yet dissipated competences in superconducting RF technologies, TESLA technology, high-level and low-level accelerator oriented RF technologies. PolFEL laser design, in cooperation with coworking international laboratories was well within the reach of the Polish accelerator R\&D community. Unfortunately the position of the relevant part of the research community is relatively low in the social hierarchy in Poland, and the design has not been accepted.

SOLARIS - recently built in the National Centre of Synchrotron Radiation at Jagiellonian University, is the first contemporary synchrotron research and user infrastructure of a bigger scale in Poland [synchrotron.uj.edu.pl]. The only big and 
pretty old, but still active, infrastructure is nuclear reactor in Swierk. User access to Solaris Poland is scheduled for 2016. Machine development is predicted to increase the number of experimental lines. Next stage of Solaris-Poland development will include extension of the infrastructure, probably with a relevant FEL, using effectively the existing accumulation ring. The ring may be then used as an injector to a superconducting liniac directly propelling the FEL undulator.

National Centre of Hadron Therapy, built at the Institute of Nuclear Physics in Krakow is now entering the commissioning period, and pre-clinical tests. The centre is built for cancer therapy. The source of high energy protons is an isochronous cyclotron. The machine is equipped in a full-dimensional scanner of the proton beam around the patient - a gantry. This was a very big infrastructure initiative of large cost, well over $50 \mathrm{M€}$.

Wider description of the possible FEL development in Poland can be found in [20-25].

\section{EUROPEAN X-RAY FEL}

Large research infrastructure, one of the biggest in the world, of the European X-ray free electron laser - EXFEL will start its life as an user facility in 2017 with a single photon line and two experimental stations. The full cost of the infrastructure is estimated for $2 \mathrm{~B} €$. Poland participates in building of the machine as a member of the EXFEL Consortium. The infrastructure consists of a laser $3,5 \mathrm{~km}$ long and a complex of research stations. The energy source is a superconducting, linear, electron accelerator with an injector of $2 \mathrm{~km}$ in length. Maximum energy of the accelerated electrons in the liniac will be initially $17,5 \mathrm{GeV}$ and next $20 \mathrm{GeV}$. Coherent, pulsed light is generated in an undulator structure using SASE mechanism. The liniac uses niobium microwave superconducting cavities of globally normalized TESLA structure, which work with a standing wave of high power and basic frequency around 1,3 $\mathrm{GHz}$ (plus third harmonic $3 \mathrm{GHz}$ ). The liniac has SRF 101 modules, with eight cavities each. The laser pulses after compression will last a few femtoseconds. Wavelength tuning is possible in the range $0,05-6 \mathrm{~nm}$. Finally the EXFEL will have five photon lines and ten experimental stations. After launching the EXFEL, at least during the next decade will have no global competitor in this domain of research X-ray sources of this large scale.

EXFEL machine of this scale gives completely new research abilities in comparison with the previous generations of light sources. A foretaste of these abilities were and are available on two, 10-times smaller, FLASH machines I and II. Around the EXFEL infrastructure, there are created official user consortia. A few of such organizations have already been accepted by the formal EXFEL organization, but the user formation and structuration process is of course still open. XBI - Integrated Biological Infrastructure - user consortium at EXFEL, coordinated by the EMBL laboratory, is building a full experimental path to investigate biological objects in the laser beam. The path includes: sample preparation, sample delivery, data quality control, and interpretation of diffraction data. SFX - Serial Femtosecond Crystallography - prepares nanocrystallography experiment standardization. The SFX experiment under construction will be an integral part of the SPB experimental line devoted to single particles, particle clusters and biomolecules. DataXpress - Analytical Hardware and Software - is a user consortium building integrated set-up of hardware and software tools for coherent diffraction experiments with single particles and nano-crystallites. CircPol user consortium - Polarized Beams - is building a set of experiments with soft X-ray pulses of controlled polarization. Polarization technique uses additional undulator interacting with the beam after its micro-bunching in the main undulator of the EXFEL machine. HIBEF user consortium - Extreme Fields - is building a series of combined hardware experiments adding to the EXFEL beam a number of high intensity optical laser beams and very high intensity pulsed magnetic fields. There will be researched dissipation phenomena in samples using combined fields. HIBEF experiments will be integrated with HED hardware - Physics of High Energy Density. HIBEF is coordinated by HZDR - Helmholtz Centrum DresdenRossendorf. User consortium COMO - Polar Molecules and Isomers - uses soft and hard X-rays to versatile molecular and cluster investigations. H-RIXS consortium carries out research with the application of the non-elastic resonant dissipation of X-rays.

\section{PHOTONIC AND ELECTRONIC INSTRUMENTS FOR FEL AND HEP}

Measurement and control apparatus, network solutions, sensors, control systems, synchronization, etc., in large experiments, in particular aspects, are usually unique for each solution. However, during the last time, due to very strong standardization of electronic hardware for big data acquisition and processing, the hardware for large experiments is following this standardization trend. Standardized are various layers of complex systems, hardware and software, including cooperation of massive data concentrators and extreme throughput peripheries. Peripheries interfaces, hardware and software measurement buses, API programming blocks, are more and more standardized and possess inbuilt intelligence. Even the most demanding and complex systems are built today of ready or only user modified functional blocks. The designers use well prepared and fast prototyped telecommunications and instrumentation standards like ATCA and $\mu$ TCA. It does not mean, however as for now, any reduction of the considerable intellectual work at the design of large control - measurement systems. The research systems always will remain a challenge for the designers. Functional requirements get more and more complex. The requirements for reliability are increasing. A complex diagnostic layer has to be inbuilt into the system, during a properly conducted co-design process, not as a simple functional overlay. The design effort shifts in the direction of building a multilayer programming environment servicing the system. This environment is also subject to evolution and standardization. The software environment works with various processors as CPU, embedded systems, DSP, FPGA, provides such functions as data transmission, acquisition and processing, assures reliability, in certain domains allows for work in the real-time, assures communications with the systems of previous generations, and many more. 
Large FEL infrastructures alone open many new research fields in biology and technology. Combination of extreme FEL beams with optical laser beams, ultra-high field pulsed magnets open yet another field hardly explored till now. It is expected that such interactions allow to have insight into subatomic structures by other method than offered by HEP technologies.

\section{REFERENCES}

[1] H.Motz, Application of the radiation from fast electron beams, J.Appl.Phys., vol.22, no.5, p.527-535 (1951)

[2] J.M.J.Madey, Stimulated emission of bremsstrahlung in a periodic magnetic field, J.Appl.Phys, vol.42, no.5 , pp.1906-1913 (1971)

[3] D.A.G.Deacon, L.R.Elias, J.M.J.Madey, G.J.Ramian, H.A.Schwettman, T.I.Smith, First operation of a free-electron laser, Phys. Rev. Lett., vol.38, no.16, p.892 (1977)

[4] R.Bonifacio, C.Pellegrini, L.M.Narducci, Collective instabilities and high-gain regime in a free electron laser, Optics Communications, vol.50, no.6, pp.373-378 (1984)

[5] G.T.Moore, The high-gain regime of the free electron laser, Nucl. Instr. and Meth, vol. A239, no.1, p.19-28 (1985);

[6] K.J.Kim and A.Sessler, "Free-electron lasers: present status and future prospects", Science vol.250, no.4977, pp.88-93 (1990)

[7] S.V.Milton, et al., "Exponential gain and saturation of a self-amplified spontaneous emission free electron laser", Science 292, 2037-2041 (2001).

[8] V.Ayvazyan, et al., "Generation of GW radiation pulses from a VUV freeelectron laser operating in the femtosecond regime", Phys. Rev. Lett. 88, 104802 (2002)

[9] G.R.Neil and L.Merminga, "Technical approaches for high-averagepower free-electron lasers", Rev. Mod. Phys. 74, 685 (2002)

[10] E.L.Saldin, E.A.Schneidmiller, M.V.Jurkov, Attosecond pulses from Xray FEL with an energy chirped electron beam and a tapered undulator, Proceedings of FEL 2006, BESSY, Berlin, Germany (2006)

[11] W.Ackermann et al., "Operation of a free-electron laser from the extreme ultraviolet to the water window", Nature Photon. 1, 336 (2007)
[12] K.Tiedtke et al., The soft x-ray free electron laser FLASH at DESY: beamlines, diagnostics and end stations, New J.Phys., vol.11, art.023029 (2009)

[13] W.A.Barletta, et al., Free electron lasers: Present status and future challenges, Nuclear Instruments and Methods in Physiucs Research Section A: Accelerators, Spectrometers, Detectors and Associated Equipment, 2010, vol.618, no.1-3, pp.69-96; doi:10.1016/j.nima.2010.02.274 (2010)

[14] P.Emma et al., "First lasing and operation of an ångstrom-wavelength free-electron laser", Nature Photon. 4, 641 (2010)

[15] J.N.Galayda et al., "X-ray free-electron lasers - present and future capabilities", JOSA B 27 (11), B106 (2010)

[16] P.Emma, et al., First lasing and operation of an angstrom-wavelength free-electon laser, Nature Photonics 4, p.641 (2010)

[17] T.Ishikawa, et al., A compact X-ray free-electron laser emitting in the subangstrom region, Nature Photonics 6, p.540-544 (2012)

[18] E.Allaria, et al., Highly coherent and stable pulses from the FERMI seeded free-electron laser in the extreme ultraviolet, Nature Photonics 6, p.699-704 (2012)

[19] Free Electron Laser research and applications [http://sbfel3.ucsb.edu/www/vl_fel.html]

[20] [20] R.S.Romaniuk, POLFEL - A Free Electron Laser in Poland, Photonics Letters of Poland, vol.1, no.3, pp.103-105 (2009) doi: 10.4302/plp.2009.3.01

[21] [21] R.S.Romaniuk, POLFEL - laser na swobodnych elektronach w Polsce, Elektronika - konstrukcje, technologie, zastosowania, vol.51, nr.4, str.83-87 (2010)

[22] [22] R.S. Romaniuk, Europejski Laser Rentgenowski, Elektronika konstrukcje, technologie, zastosowania, vol.54, nr.4, str 149-154 (2013)

[23] [23] R.S.Romaniuk, Lasery rentgenowskie LCLS i LCLS II: SLAC, Elektronika - konstrukcje, technologie, zastosowania, vol54, nr.5, str.6669 (2013)

[24] R.S.Romaniuk, Rozwój laserów na swobodnych elektronach, Elektronika, vol.57, no.3, pp.16-21, 2016

[25] R.S.Romaniuk, Źródła światła piątej generacji, Elektronika, vol.57, no.4 pp. $42-50,2016$ 